Results: The median volumes of GTV and CTV were $39.5 \mathrm{cc}(0.8-158.9 \mathrm{cc})$ and $112.7 \mathrm{cc}(32.0-338.9 \mathrm{cc})$ respectively. The treatment plans showed that the D95 and V95 of GTV were $66.9 \mathrm{~Gy}$ and $98.3 \%$, respectively. The minimum, maximum and mean dose to GTV was $54.8 \mathrm{~Gy}, 77.1 \mathrm{~Gy}$ and $69.8 \mathrm{~Gy}$. The mean fractional dose was $2.32 \mathrm{~Gy}(1.99-2.91 \mathrm{~Gy})$. The average doses of the surrounding critical structures were much lower than the tolerance thresholds. Median follow-up was 12 months (range, 2-47 months). The 1-, 2- and 3-year local progression-free and overall survival rates were $96.4 \%, 88.4 \%, 85.3 \%$, and $65.9 \%$, $49.6 \%, 41.6 \%$, respectively. Eleven patients $(8.3 \%)$ developed distant metastases. On univariate analysis, T stage $(\mathrm{p}=0.009)$, maximum reirradiation dose to GTV $(p=0.006)$, fractional dose to GTV $(p=0.02)$, the volume of GTV and CTV $(p=0.003 \&$ 0.004), were significant prognostic factors for overall survival. On multivariate analysis by Cox proportional hazard model, only fractional dose $(p=0.029)$ and the volume of GTV and CTV $(p=0.000)$ remained significant for overall survival.

Conclusions: Reirradiation using IMRT may improve the local control of the local recurrence and prolong survival of these patients. The fractional dose was delivered to GTV and the volume of GTV and CTV were independent prognostic factors for overall survival.

\title{
1007 Longitudinal Changes in Quality of Life and Treatment Costs in Long-term Survivors of Tumors of the Oropharynx Treated with Brachytherapy or Surgery
}

\author{
P.C. Levendag, ${ }^{1}$ W. Nijdam, ${ }^{1}$ I. Noever,${ }^{1}$ M. Agthoven, van, ${ }^{2}$ P. Schmitz, ${ }^{1}$ C. Uyl-de Groot ${ }^{2}$ \\ ${ }^{1}$ Radiation Oncology, Erasmus MC - Daniel den Hoed, Rotterdam, Netherlands, ${ }^{2}$ Institute for Medical Technology \\ Assessment, Rotterdam, Netherlands
}

Purpose/Objective: To assess the longitudinal changes in quality of life (QOL) and associated treatment costs in long-term survivors of oropharyngeal cancers treated with either brachytherapy (BT) or surgery (S).

Materials/Methods: From 1991-2001, 144 patients with tonsillar fossa (TF) and/or soft palate (SP) tumors, and cancers of the base of tongue (BOT), were treated by organ function preservation, using external beam radiotherapy (EBRT) and BT. 110 patients not suitable for BT were treated with a combined resection with postoperative EBRT. Details of the RT techniques and clinical results have been published previously (IJROBPh 2004;59:713-724). Among all patients $>2$ and $<10$ years alive and NED, a QOL survey was conducted in 2003, studying 3 groups: group I with TF/SP tumors treated by S (41), group II with TF/SP tumors treated by BT (57), and group III consisting of BOT cancers treated by BT (18). The object was to determine the performance status scales (PSS) scores according to List et al. (Cancer 1990; 66:564-569); i.e. the PSS for eating in public (EP), understandability of speech (US), and normalcy of diet (ND). In addition, symptoms related to xerostomia (dry mouth, DM) and the (in)ability to swallow (drink to eat, DE), were measured by standardized queries and a visual analogue scale (VASxero).

In 2005, the PSS survey was repeated among same NED patients. All questionnaires returned within 1 month were eligible for analysis (response 96\%). The questionnaires of both surveys were returned by 22 patients of group I, 24 of group II, and 3 of group III. By regression analysis, the effect of time from diagnosis, age, dose, sex, T-stage, N-stage, trismus, and necrosis (ulcer) on the PSS scores was determined. As part of the 2005 survey, the EORTC QLQ-C30, EORTC H\&N35, and Euroqol (EQ5D) questionnaires were sent concurrently with the 2nd PSS mailing. The mean scores were calculated as specified in the EORTC manuals. For each treatment group treatment costs were computed.

Results: In both surveys, the mean PSS scores as well as the scores for the queries related to xerostomia were not significantly different. The mean value for all BT patients versus all S patients were 34 vs. $42(p=0.40)$ for $D M, 39$ vs. $48(p=0.38)$ for $D E$, 38 vs. $46(\mathrm{p}=0.36)$ for VASxero, 52 vs. $67(\mathrm{p}=0.27)$ for $\mathrm{EP}, 75$ vs. $93(\mathrm{p}=0.009)$ for US and 61 vs. $70(\mathrm{p}=0,41)$ for ND.

QLQ-C30 (general health related QOL): no significant differences between patients treated by S or BT. QLQ-H\&N35 (head $\&$ neck cancer specific QOL): BT patients experience significantly more pain $(\mathrm{p}=0.05), \mathrm{S}$ patients more problems with opening mouth $(\mathrm{p}=0.02)$. EQ5D (functional and symptom items): no distinction between the groups. Mean costs per group: I \$ 35.755 , II \$ 19.555 , and III \$ 26.630 .

Conclusions: Traditionally tumor control and survival have been the primary endpoints in evaluating $\mathrm{H} \& \mathrm{~N}$ cancer treatment. Health-related QOL and treatment costs are now increasingly being used to evaluate treatment modalities. The QOL surveys show that item for item the mean PSS scores were not significantly different for the 3 groups in 2003 as opposed to 2005; moreover, they did not change in time. For each group, discriminating factors seem to be modality related rather than site specific (e.g. BT more ulceration, surgery more trismus, and for both modalities the dry mouth syndrome). Due to the number of admission days, S is more expensive as to BT. Given the good localregional control rates for BT and S (85\% at 10-years), the data suggest that taking into account other parameters, such as (functional) QOL and associated costs, can be of additional value when discriminating between treatment modalities.

\section{Evaluation of Larynx Dose with Extended Field IMRT for Head and Neck Cancer}

\author{
L.E. Millender, M.K. Bucci, Y. Kim, J. Quivey, P. Xia \\ Radiation Oncology, University of California San Francisco, San Francisco, CA
}

Purpose/Objective: To determine the radiation dose to the larynx with extended field IMRT (EF-IMRT) and to compare tumor and larynx doses obtained with EF-IMRT and a conventional split beam technique.

Materials/Methods: Twenty patients with node positive oropharyngeal or nasopharyngeal head and neck cancer were selected for this study. All patients were treated with EF-IMRT plans that encompassed the primary tumor, neck nodes, and supraclavicular regions. The treatment goal was to deliver a dose of $70 \mathrm{~Gy}$ to $>95 \%$ of the planning gross tumor volume (pGTV), $59.4 \mathrm{~Gy}$ to the planning clinical tumor volume (pCTV), and 54 Gy to the planning elective tumor volume (pETV). Although a larynx block was contoured for each patient during EF-IMRT planning the size was not uniform. For this study the entire larynx was re-contoured including all soft tissue, muscles, and cartilage from the superior thyroid cartilage to the inferior cricoid cartilage. Dose to the whole organ was retrospectively determined. Conventional split beam AP supraclavicular field plans were also generated. Doses to the larynx, pGTV, pCTV, and pETV were determined and compared to doses obtained with EF-IMRT treatment of the same volumes. 